\title{
A FOLLOW-UP NOTE ON THE PLAUSIBILITY OF THE LEONTIEF AND GHOSH CLOSED MODELS.
}

\author{
ANTONIO MANRESA ${ }^{\mathrm{a}^{*}}$ and FERRAN SANCHO \\ ${ }^{\text {aDepartment }}$ of Economics and CREB, Universitat de Barcelona, Spain \\ bepartment of Economics, Universitat Autònoma de Barcelona, Bellaterra, Spain
}

*Corresponding author. Email: manresa@ub.edu

\begin{abstract}
We consider in this note the closed models of Leontief and Ghosh that make endogenous, respectively, part of final demand and part of value-added. We take the numerical three-sector example of Oosterhaven (2012) to show that anomalies of the sort he finds for the Ghosh closed model, which was introduced by Guerra and Sancho (2011), can also be found in the closed version of Leontief's model. Under the assumption that aggregate exogenous resources are fixed, Oosterhaven shows that in the closed Ghosh model less value-added may go hand in hand with more output. Under the same type of assumption, we also show that in the Leontief closed model less final demand may also go hand in hand with more output. These numerical anomalous results for the three-sector case turn out to be generic in both closed models for any $2 \times 2$ Input-Output model but a general proof for the $n \times n$ case is still lacking.
\end{abstract}

Keywords: demand-driven model, supply-driven model, closed linear models. 


\section{Introduction}

Guerra and Sancho (2011) extend Ghosh's model to include the income feedbacks between valueadded and consumption demand in a manner which is, mathematically speaking, fully equivalent to Leontief's closed model when private consumption is made endogenous. In Guerra and Sancho (2011) value-added is seen to grow in all sectors along with their output, which overcomes the usual implausibility found in such model. In turn, Oosterhaven (2012) uses the same numerical $3 \times 3$ input-output table from Guerra and Sancho (2011) and introduces an additional rule in the exogenous variables (i.e. value-added) of the closed Ghosh model. This rule consists in performing a neutral redistribution among sectors of the fixed exogenous aggregate resources, which we can interpret as the implementation of some policy directive. Oosterhaven (2012) solves the model under the neutral redistribution and finds an inverse relationship between value-added and output: less value-added may go hand in hand with more output, both at the sectorial and aggregate levels. From this result, Oosterhaven (2012) concludes that the closed model is even more implausible than the standard Ghosh model.

The purpose of this note is to show that the same type of anomalous economic behavior that affects the Ghosh closed also affects the Leontief closed model. We also use a 3x3 input-output table, apply the same neutral redistribution rule to Leontief's closed model - in this case to final demand - and end up observing an inverse relationship between output and endogenous consumption demand, at the sectorial and aggregate levels. That is, less final demand in the economy may also go hand by hand with more output. We may also refer to this mismatch between changes in demand and changes in output as an alleged anomaly, or implausible behavior, of the Leontief closed model.

These findings are not surprising if we take into account that both models, once we close them in relation to households' final demand and value-added ownership, share a common mathematical structure. In fact, in Manresa and Sancho (2013) we show that these alleged anomalies of both models, under the neutrality rule, will always happen in a $2 \times 2$ sector Input-Output framework, except in very special cases of the base input-output data that are quite unlikely in empirical terms ${ }^{1}$. If we categorize these models' behavior as implausible or anomalous, this concern requires further scrutiny, and this is what we do in this short note. We believe it is important for input-output researchers and practitioners to be aware of the possibilities, limitations and issues that may affect the models we so much cherish and use. Inasmuch as we keep using these models, we feel it is essential to know and report all their properties, positive or negative. In Section 2 we briefly describe the main features of both extended models. In Section 3 we discuss the nature and the implications of some numerical examples to shed some light on this issue. Section 4 ends the note with some general concluding remarks.

\footnotetext{
${ }^{1}$ In short, all sectors must share the same ratio of consumption demand to output for Leontief's model, or domestic value-added to output for Ghosh's model.
} 


\section{Brief conceptual reminder of the closed Leontief and Ghosh models.}

Let us start from an $n$ sector input-output economy described by observed data $(\mathbf{x}, \mathbf{f}, \mathbf{v}, \mathbf{Z})$ where vector $\mathbf{x}$ stands for gross output, vector $\mathbf{f}$ is final demand, vector $\mathbf{v}$ is value-added, and matrix $\mathbf{Z}$ stands for intermediate transactions ${ }^{2}$. Using the data and assuming the usual proportionality relationships, we define technical and sales coefficients matrices $\mathbf{A}=\left(a_{i j}\right)$ and $\mathbf{B}=\left(b_{i j}\right)$, respectively, by way of:

$$
\begin{aligned}
& a_{i j}=z_{i j} / x_{j} \\
& b_{i j}=z_{i j} / x_{i}
\end{aligned}
$$

In the simple Leontief model matrix $\mathbf{A}$ links final demand $\mathbf{f}$ with output $\mathbf{x}$ through the linear relationship:

$$
\mathbf{x}=(\mathbf{I}-\mathbf{A})^{-1} \mathbf{f}=\Phi_{L}(\mathbf{f})
$$

Similarly, in the simple Ghosh model we would have matrix B linking value-added $\mathbf{v}$ with output $\mathbf{x}$ by:

$$
\mathbf{x}^{\prime}=\mathbf{v}^{\prime}(\mathbf{I}-\mathbf{B})^{-1}=\Phi_{G}\left(\mathbf{v}^{\prime}\right)
$$

Notice that value-added $\mathbf{v}$ is not present in expression (2) and hence plays no role in output determination; similarly for $\mathbf{f}$ in (3). This endogeneity gap can be closed through the closed models ${ }^{3}$. However, since no economic model can be completely closed $^{4}$, we will distinguish within $\mathbf{f}$ and $\mathbf{v}$ an endogenous part and an exogenous remainder, say 'private' (endogenous) and 'public' (exogenous) final demand and value-added. Therefore, we make $\mathbf{f}=\mathbf{f}_{1}+\mathbf{f}_{2}$, and $\mathbf{v}=\mathbf{v}_{1}+\mathbf{v}_{2}$, with index ' 1 ' standing for 'private' and ' 2 ' for 'public'. In Leontief's closed model, private demand $\mathbf{f}_{1}$ is made endogenous in response to changes in $\mathbf{v}$ triggered by changes in $\mathbf{x}$, whereas in the Ghosh closed model $\mathbf{v}_{1}$ is made endogenous and dependent on changes in $\mathbf{f}$, which are in turn triggered by any changes happening in $\mathbf{x}$. Let us denote these relationships by $\mathbf{f}_{1}=\psi_{L}(\mathbf{v}(\mathbf{x}))$ in Leontief, and $\mathbf{v}_{1}^{\prime}=\psi_{G}\left(\mathbf{f}\left(\mathbf{x}^{\prime}\right)\right)$ in Ghosh. Substituting them in (2) and (3) we obtain:

$$
\mathbf{x}=\Phi_{L}(\mathbf{d})=\Phi_{L}\left(\mathbf{f}_{1}+\mathbf{f}_{2}\right)=\Phi_{L}\left(\psi_{L}(\mathbf{v}(\mathbf{x}))+\mathbf{f}_{2}\right)
$$

\footnotetext{
${ }^{2}$ The notational convention is that $\mathbf{y}$ represents a column vector and $\mathbf{y}^{\prime}$ a row vector.

${ }^{3}$ See Leontief $(1941,1953)$ for a description of the closed model.

${ }^{4}$ Leontief (1986) concludes that we cannot truly close static models, hence the need for some exogenous driving force such as investment, government demand or net exports.
} 


$$
\mathbf{x}^{\prime}=\Phi_{G}\left(\mathbf{v}^{\prime}\right)=\Phi_{G}\left(\mathbf{v}_{1}^{\prime}+\mathbf{v}_{2}^{\prime}\right)=\Phi_{G}\left(\psi_{G}\left(\mathbf{f}\left(\mathbf{x}^{\prime}\right)\right)+\mathbf{v}_{2}^{\prime}\right)
$$

We can solve these two expressions for equilibrium solutions in both closed models and compare initial and counterfactual allocations.

\subsection{Oosterhaven's neutrality rule}

For the Ghosh closed model Guerra and Sancho (2011) show that changes in the exogenous part of value-added imply not only that output increases everywhere - as expected - but that total value-added increases in all sectors as well, thus breaking the implausibility of the simple Ghosh model regarding unresponsive value-added to changes in output. In differential terms this result could be expressed as:

$$
\Delta v_{k, 2}>0 \text { implies } \forall i \Delta x_{i} \geq 0 \text { and } \forall i \Delta v_{i} \geq 0
$$

That is, any positive exogenous change in sector $k$ value-added will generate non-negative increases in output and total value-added in all sectors.

In the example of Oosterhaven (2012) for the Ghosh model, the use of the neutral reshuffling rule keeps constant the level of aggregate exogenous value-added. He takes two sectors, say sectors $n$ and $m$, and assumes that sector $n$ has 5 less units of the exogenous resource whereas sector $m$ has 5 more units. Total use is therefore constant in the aggregate. Notice the possible policy interpretation of these changes. A direct application of Guerra and Sancho's (2011) result from expression (4) yields:

$$
\begin{aligned}
& \Delta v_{n, 2}=-5 \text { implies } \forall i \Delta x_{i} \leq 0 \text { and } \forall i \Delta v_{i} \leq 0 \\
& \Delta v_{m, 2}=+5 \text { implies } \forall i \Delta x_{i} \geq 0 \text { and } \forall i \Delta v_{i} \geq 0
\end{aligned}
$$

If we add up the individually correct implications for sectors $n$ and $m$, we can check that the overall combined effects do not have any definite sign; anything goes. Observe, however, that all that is taking place here is the summation (thanks to the separability implicit in the linear models) of two independent general equilibrium adjustments that work in the opposite direction of each other. The implausible behavior that Oosterhaven (2012) describes comes from the interplay, within the structure of the linear model, of two equilibrium shocks working in opposite directions under the constraint of constant aggregate resources.

The same would occur verbatim in the Leontief extended model. It is mathematically straightforward to derive the counterpart of Property (4) for the Leontief closed model version:

$$
\Delta f_{k, 2}>0 \text { implies } \forall i \Delta x_{i} \geq 0 \text { and } \forall i \Delta f_{i} \geq 0
$$

For the same type of neutral rule, if we apply it to the exogenous part of final demand in sectors $n$ and $m$ and we use identical values as above we will now conclude:

$$
\begin{aligned}
& \Delta f_{n, 2}=-5 \text { implies } \forall i \Delta x_{i} \leq 0 \text { and } \forall i \Delta f_{i} \leq 0 \\
& \Delta f_{n, 2}=+5 \text { implies } \forall i \Delta x_{i} \geq 0 \text { and } \forall i \Delta f_{i} \geq 0
\end{aligned}
$$


Notice from expression ( $\left.5^{\prime}\right)$ that, once again, the application of the neutral rule can give rise, $a$ priori, to any sign in $\Delta x_{i}$ and in $\Delta \mathrm{f}_{i}$. As long as signs are concerned, therefore, we may expect that the same type of implausible behavior of the endogenous variables could take place in the close Leontief model — and for the exact same formal reasons — as in the closed Ghosh model.

We now move to the numerical examples constructed using the neutrality rule.

\section{Numerical examples based on the Guerra and Sancho I-O table and its transposed}

The highlights of this section are that, under the neutrality rule, we will observe in the Ghosh closed model that an increase in total production in one sector goes along with a decrease in its total value-added. Similarly, we will observe in the Leontief closed model sectors where an increase in total production is accompanied by a decrease in final demand.

\subsection{Implausible behavior in the Ghosh closed model.}

Using Guerra and Sancho (2011) numerical Input-Output table (see the Appendix), Oosterhaven (2012, page 107, Table 2) calculates his results, which we reproduce as Table 1 here. These numerical results lay down the foundations of his criticism to the closed Ghosh model. The results of a change on production, $\Delta \mathbf{x}$, value-added, $\Delta \mathbf{v}$, and final demand, $\Delta \mathbf{f}$, stem from the implementation of the neutral resource shift in two of the sectors. He concludes that the equilibrium magnitudes of the closed Ghosh model present an anomalous behavior. Namely, that output and value-added move in different directions. For instance, the addition of five units of the public input in sector 2 together with a reduction of five units in sector 3 yields Oosterhaven's last block of columns in Table 1. In this block of numbers, we clearly see that at the sectorial level, more output goes along with less value-added in sectors 1 ( +0.63 vs. -0.17$)$ and $2(+4.82$ vs. -0.09$)$, and the same happens at the aggregate level $(+1.92$ vs. -0.60$)$. From this particular anomaly in this simulation, Oosterhaven (2012) concludes that the closed model of Ghosh is even more implausible than the simple Ghosh model.

[Table 1 around here]

\subsection{Implausible behavior in the Leontief closed model.}

Following the same experiment performed by Oosterhaven for the Ghosh closed model, we now focus on our symmetrical reasoning as applied to Leontief's closed model. We build the results in Table 2 using the transposed of the input-output table of Guerra and Sancho (2011) (see the Appendix). We then use the same neutral redistribution rules as before and we obtain (by simple duality) the same numbers as in Table 1 with no need for actual new computations.

Looking at the second block of numbers in Table 2 (from the left) gives us the Leontief counterpart to the data for Ghosh in Table 1. We can see the same numbers as in the Ghosh case, simply because of the transposition of the I-O table, but they are now in two different columns. We see that final demand for goods 1 and 2 goes down $(-0.17$ and -0.09$)$ whereas their respective sectorial output goes up $(+0.63$ and +4.82$)$. Even worse, total output increases $(+1.92)$ even though aggregate demand goes overall down $(-0.60)$, along with the same happening in aggregate value- 
added $(-0.60)$. There is less demand for goods in general $(-0.60)$ and less value-added $(-0.60)$ around but, even so, the economy ends up producing more goods $(+1.92)$. The behavior of the aggregate variables in the Leontief closed model mimics what we observe happens as well in Ghosh's closed model. For the same reasons, we may therefore conclude that such behavior is implausible as well.

\section{[Table 2 around here]}

It is also worth remarking that the aggregate behavior of Ghosh's closed model under this new transposed I-O table is completely regular under both simulations. For example, when exogenous value-added is increased in 5 units in sector 1 and reduced in 5 units in sector 3, aggregate output, aggregate value-added and aggregate final demand all go down $(-2.70,-1.54,-1.54)$. In other words, if Guerra and Sancho (2011) had used what we call here the transposed I-O table, it would have not been possible to find any anomalous aggregate behavior in the closed Ghosh model. The aggregate anomaly, in this case, would have appeared in the Leontief model — and only in the Leontief model.

Finally, the aggregate variables of the closed Leontief model would behave in the proper way when implemented using the data in Guerra and Sancho (2011) original I-O table. When we look at the sectoral variables, we observe that in all eight simulations in Tables 1 and 2 there is always a sector where output and value-added move in the opposite direction in the Ghosh model and the same occurs with output and final demand in the Leontief model. These numerical examples are in accord with the general results reported in Manresa and Sancho (2013) for two sector economies ${ }^{5}$.

\section{Final remarks}

Remark 1. We show that the anomalous economic behavior reported by Oosterhaven (2012) for the closed Ghosh model has a parallel counterpart in the Leontief closed model. In the model of Ghosh, we see that more aggregate, or sectoral, output may go hand in hand with less value-added. Correspondingly, in the closed Leontief model we find that a higher level of aggregate, or sectoral, output may go along with less final demand. The question is if this behavior happens by chance and it is just a reflection of a particular configuration of the I-O data. The tentative answer seems to be no, as the general results for the $2 \times 2$ input-output case shows and the $3 \times 3$ examples discussed here suggest. For the $n \times n$ general I-O framework, we need to develop further research either by generalizing (or eventually disproving) the $2 \times 2$ results, or else by systematic numerical simulations that would allow to ascertain the nature and prevalence of these types of effects.

Remark 2. These results, however provisional, give us a warning since they pinpoint some of the limitations of the research that uses these models either for theoretical or empirical purposes. Of particular relevance are the possible consequences for public policy design. Think of the government implementing a new policy under some aggregate resource restriction, like redirecting public expenditure from sector $n$ to sector $m$ under a fixed budget constraint, for whatever reason.

\footnotetext{
${ }^{5}$ In fact, Manresa and Sancho (2013) formally demonstrate in a $2 \times 2$ linear model that these types of regularities are generic in the equilibrium solution of the two closed models.
} 
Our results as well as Oosterhaven's indicate that policy makers should be aware of the unintended and possible perverse outcomes, contrary to the purported policy goals, that may ensue.

Acknowledgments. Support from projects ECO2016-75204P (first author), and MICINNECO2017-83534P (second author) is gratefully acknowledged. We are also grateful to a referee, the editors Bart Los and Michael Lahr, and to the participants of the Santiago de Compostela I-O Network meeting of 2018 for their valuable comments.

TABLE 1. Oosterhaven's (2012) results with two decimal digits

\begin{tabular}{|c|c|c|c|c|c|c|c|c|c|c|c|c|}
\hline \multirow[b]{2}{*}{ Scenario } & \multicolumn{6}{|c|}{ Extended Leontief model } & \multicolumn{6}{|c|}{ Extended Ghosh model } \\
\hline & \multicolumn{3}{|c|}{$(+5$ in $1 \&-5$ in 3$)$} & \multicolumn{3}{|c|}{$(+5$ in $2 \&-5$ in 3$)$} & \multicolumn{3}{|c|}{$(+5$ in $1 \&-5$ in 3$)$} & \multicolumn{3}{|c|}{$(+5$ in $2 \&-5$ in 3$)$} \\
\hline Sector & $\Delta \mathbf{x}$ & $\Delta \mathbf{v}$ & $\Delta d$ & $\Delta \mathbf{x}$ & $\Delta \mathbf{v}$ & $\Delta \mathbf{d}$ & $\Delta \mathbf{x}$ & $\Delta \mathbf{v}$ & $\Delta \mathbf{d}$ & $\Delta \mathbf{x}$ & $\Delta \mathbf{v}$ & $\Delta \mathbf{d}$ \\
\hline 1 & +4.75 & +0.95 & -0.77 & -2.35 & -0.47 & -1.41 & +8.20 & +0.64 & +2.87 & +0.63 & -0.17 & +0.22 \\
\hline 2 & -1.65 & -0.17 & -0.11 & +1.95 & +0.20 & -0.20 & +1.66 & +0.32 & +0.09 & +4.82 & -0.09 & +0.24 \\
\hline 3 & -5.80 & -2.32 & -0.67 & -6.40 & -2.55 & -1.21 & -2.36 & +1.29 & -0.71 & -3.53 & -0.34 & -1.06 \\
\hline Total & -2.70 & -1.54 & -1.54 & -6.75 & -2.82 & -2.82 & +7.50 & +2.25 & +2.25 & +1.92 & -0.60 & -0.60 \\
\hline
\end{tabular}

Source: Oosterhaven (2012), Table 2, pp.107, built from the given I-O data in Guerra and Sancho (2011).

TABLE 2. Output, value-added and final demand for the two I-O models, same scenarios as in Oosterhaven (2012)

\begin{tabular}{|c|c|c|c|c|c|c|c|c|c|c|c|c|}
\hline \multirow[b]{2}{*}{ Scenario } & \multicolumn{6}{|c|}{ Extended Leontief model } & \multicolumn{6}{|c|}{ Extended Ghosh model } \\
\hline & \multicolumn{3}{|c|}{$(+5$ in $1 \&-5$ in 3$)$} & \multicolumn{3}{|c|}{$(+5$ in $2 \&-5$ in 3$)$} & \multicolumn{3}{|c|}{$(+5$ in $1 \&-5$ in 3$)$} & \multicolumn{3}{|c|}{$(+5$ in $2 \&-5$ in 3$)$} \\
\hline Sector & $\Delta \mathbf{x}$ & $\Delta \mathbf{v}$ & $\Delta \mathbf{d}$ & $\Delta \mathbf{x}$ & $\Delta \mathbf{v}$ & $\Delta \mathbf{d}$ & $\Delta \mathbf{x}$ & $\Delta \mathbf{v}$ & $\Delta d$ & $\Delta \mathbf{x}$ & $\Delta \mathbf{v}$ & $\Delta \mathbf{d}$ \\
\hline 1 & +8.20 & +2.87 & +0.64 & +0.63 & +0.22 & -0.17 & +4.75 & -0.77 & +0.95 & -2.35 & -1.41 & -0.47 \\
\hline 2 & +1.66 & +0.09 & +0.32 & +4.82 & +0.24 & -0.09 & -1.65 & -0.11 & -0.17 & +1.95 & -0.20 & +0.20 \\
\hline 3 & -2.36 & -0.71 & +1.29 & -3.53 & -1.06 & -0.34 & -5.80 & -0.67 & -2.32 & -6.40 & -1.21 & -2.55 \\
\hline Total & +7.50 & +2.25 & +2.25 & +1.92 & -0.60 & -0.60 & -2.70 & -1.54 & -1.54 & -6.75 & -2.82 & -2.82 \\
\hline
\end{tabular}

Source: From "transposing" the I-O data from Table 2a in Guerra and Sancho (2011).

\section{References}


Ghosh, A. (1958), Input-output approach in an allocation system. Economica, 25, pp. 58-64.

Guerra, A.I. and F. Sancho (2011), Revisiting the original Ghosh model: can it be made more plausible?. Economic Systems Research, vol. 23(3), pp. 319-328.

Leontief, W. (1936), Quantitative input and output relations in the economic system of the United States. Review of Economics and Statistics, 28, pp. 105-25.

Leontief, W. (1941), The Structure of the American Economy 1919-1939. New York: Oxford University Press.

Leontief, W. (1953), Studies in the Structure of the American Economy. New York: Oxford University Press.

Leontief, W. (1986), Input-output economics. New York: Oxford University Press (second edition).

Manresa, A. and F. Sancho (2013), Supply and demand biases in linear interindustry models. Economic Modelling, vol. 33, pp. 94-100.

Oosterhaven, J. (2012), Adding supply-driven consumption makes the Ghosh model even more implausible, Economic Systems Research, vol. 24(1), pp. 101-111.

\begin{tabular}{|c|c|c|c|c|c|c|}
\hline \multicolumn{6}{|c|}{ Appendix: the original and transposed I-O tables } & \\
\hline \multicolumn{5}{|c|}{ Input-output table from Guerra and Sancho (2011) } & \multirow[b]{2}{*}{ Collective } & \multirow[b]{2}{*}{ Tota } \\
\hline & Sector 1 & Sector 2 & Sector 3 & Private Agent & & \\
\hline Sector 1 & 30 & 20 & 10 & 35 & 5 & 100 \\
\hline Sector 2 & 20 & 10 & 40 & 5 & 25 & 100 \\
\hline Sector 3 & 10 & 20 & 5 & 30 & 35 & 100 \\
\hline Value-added & 20 & 10 & 40 & & & 70 \\
\hline Collective & 20 & 40 & 5 & & & 65 \\
\hline Total & 100 & 100 & 100 & 70 & 65 & \\
\hline & & & & & & \\
\hline \multirow{2}{*}{\multicolumn{4}{|c|}{ The transposed input-output table }} & & & \\
\hline & & & & & & \\
\hline & Sector 1 & Sector 2 & Sector 3 & Private Agent & Collective & Total \\
\hline Sector 1 & 30 & 20 & 10 & 20 & 20 & 100 \\
\hline Sector 2 & 20 & 10 & 20 & 10 & 40 & 100 \\
\hline Sector 3 & 10 & 40 & 5 & 40 & 5 & 100 \\
\hline Value-added & 35 & 5 & 30 & & & 70 \\
\hline Collective & 5 & 25 & 35 & & & 65 \\
\hline Total & 100 & 100 & 100 & 70 & 65 & \\
\hline
\end{tabular}

